\title{
An epigenetic switch governing daughter cell separation in Bacillus subtilis
}

\author{
Yunrong Chai, ${ }^{1}$ Thomas Norman, ${ }^{1}$ Roberto Kolter, ${ }^{2}$ and Richard Losick ${ }^{1,3}$ \\ ${ }^{1}$ Department of Molecular and Cellular Biology, Harvard University, Cambridge, Massachusetts 02138, USA; ${ }^{2}$ Department \\ of Microbiology and Molecular Genetics, Harvard Medical School, Boston, Massachusetts 02115, USA
}

\begin{abstract}
Growing cells of Bacillus subtilis are a bistable mixture of individual motile cells in which genes for daughter cell separation and motility are ON, and chains of sessile cells in which these genes are OFF. How this ON/OFF switch is controlled has been mysterious. Here we report that a complex of the SinR and SlrR proteins binds to and represses genes involved in cell separation and motility. We also report that SinR and SlrR constitute a doublenegative feedback loop in which SinR represses the gene for SlrR (sIrR), and, by binding to (titrating) SinR, SlrR prevents SinR from repressing slrR. Thus, SlrR indirectly derepresses its own gene, creating a self-reinforcing loop. Finally, we show that, once activated, the loop remains locked in a high SlrR state in which cell separation and motility genes are OFF for extended periods of time. SinR and SlrR constitute an epigenetic switch for controlling genes involved in cell separation and motility.
\end{abstract}

[Keywords: Epigenetic; bistability; biofilm; Bacillus subtilis]

Supplemental material is available at http://www.genesdev.org.

Received February 9, 2010; revised version accepted March 2, 2010.

The bacterial cell cycle involves mechanisms for dividing cells in two (binary fission), and for segregating copies of the genome to each daughter cell (Bramhill 1997; Goehring and Beckwith 2005; Margolin 2005). In certain bacteria, such as Bacillus subtilis, the cell cycle involves an additional, regulated step: the separation of daughter cells from each other by cleavage of the cell wall (peptidoglycan) that binds them together following cytokinesis (Blackman et al. 1998; Smith et al. 2000). Binary fission in B. subtilis is mediated by the formation of a division septum at mid-cell that arises by constriction of the cytoplasmic membrane and the inward growth of the peptidoglycan to form a disc-like partition between the resulting daughter cells (Errington et al. 2003). Cell wall hydrolytic enzymes called autolysins subsequently cleave the peptidoglycan across the long axis of the septum separating the daughter cells from the other (Smith et al. 2000). In B. subtilis, daughter cell separation is regulated in that, under some circumstances, cells remain linked together through multiple rounds of binary fission, forming long chains (Branda et al. 2001; Kearns and Losick 2005). However, the mechanism that controls cell separation has remained largely mysterious. Here we report the discovery of an epigenetic switch composed of a double-negative feedback loop that regulates the production of the autolysins that mediate cell separation.

${ }^{3}$ Corresponding author.

E-MAIL losick@mcb.harvard.edu; FAX (617) 496-4642.

Article published online ahead of print. Article and publication date are online at http://www.genesdev.org/cgi/doi/10.1101/gad.1915010.
One circumstance in which chains are observed is in the mid-exponential phase of growth, when the population is a mixture of two cell types: individual motile cells, and long chains of sessile cells (Kearns and Losick 2005). Autolysin genes and motility genes are transcribed by RNA polymerase containing the alternative $\sigma$ factor $\sigma^{D}$ (Helmann et al. 1988; Mirel and Chamberlin 1989; Marquez et al. 1990; Kuroda and Sekiguchi 1993; Mirel et al. 1994; Margot et al. 1999; Chen et al. 2009). Cells in which these genes are ON are motile, and cells in which these genes are OFF form long chains of sessile cells. That cells in the exponential phase of growth can persist in ON or OFF states through multiple rounds of division has suggested that autolysin and motility genes are subject to a bistable switch (Kearns and Losick 2005; Cozy and Kearns 2010). It was speculated that the bistable switch is a form of bet hedging in which the swimmers (nomadic cells) seek new sources of nutrients while the sessile chains exploit an existing niche.

A second circumstance in which chaining is observed is in structured communities called biofilms, which can form on solid surfaces as wrinkled colonies or at the air/liquid interface of standing cultures as floating pellicles (Branda et al. 2001; Kobayashi 2007; Vlamakis et al. 2008; López et al. 2009). Biofilms consist of long chains of cells that are attached to each other along their long axes by an extracellular matrix (Branda et al. 2001; Kobayashi 2007). Chaining in the context of the biofilm can be understood as facilitating the bundling of cells together by the matrix. 
Biofilm formation is governed by a regulatory circuit that involves three dedicated regulatory proteins: SinI, SinR, and SlrR (Kearns et al. 2005; Chu et al. 2008; Kobayashi 2008). SinI is produced under the control of Spo0A, a global regulator of post-exponential-phase gene expression in B. subtilis (Gaur et al. 1988; Sonenshein 2000; Piggot and Hilbert 2004; Fujita et al. 2005; Chai et al. 2008). SinI is an anti-repressor that binds to and inhibits the SinR repressor, a protein that is produced constitutively (Bai et al. 1993; Kearns et al. 2005). SinR, in turn, binds to the promoters for matrix operons (yqxM and eps [exopolysaccharide]) and the gene for SlrR (slrR), blocking their transcription (Fig. 1A; Kearns et al. 2005; Branda et al. 2006; Chu et al. 2006, 2008). SlrR is a hybrid protein, containing domains that are homologous to both the SinI anti-repressor and the SinR repressor (Chu et al. 2008).

We report that SinR and SlrR constitute a doublenegative feedback loop involving protein-protein (SlrRmediated inhibition of SinR) and protein-DNA (SinRmediated repression of $\operatorname{sir} R$ ) interactions. We show that SlrR binds to SinR, and that the formation of the SinR $\bullet$ SlrR complex inhibits the capacity of SinR to bind

A

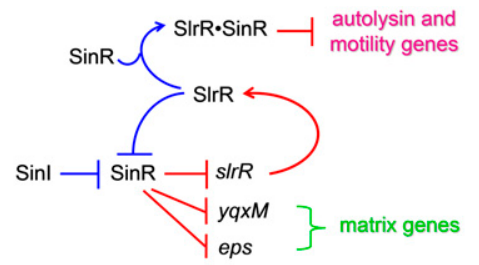

B

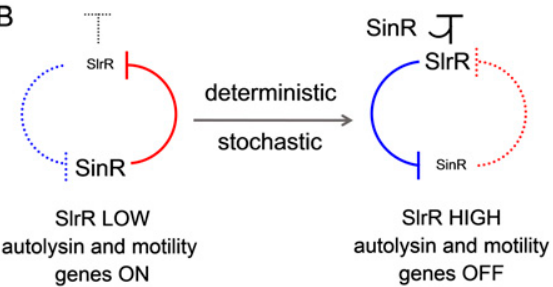

Figure 1. The SinR SlrR switch. (A) Genetic circuit controlling matrix production and cell chaining. SinI is an anti-repressor that inhibits the SinR repressor, thereby derepressing the matrix operons (yqxM and eps) and the gene for SlrR. The resulting synthesis of SlrR has two consequences. It leads to the formation of the SlrR・SinR heterocomplex, which represses autolysin and motility genes. Synthesis of SlrR also leads to inhibition of SinR (through titration of SinR), thereby further derepressing slrR as well as the matrix operons. (B) SinR and SlrR constitute a double-negative loop that operates at the level of proteinprotein interaction (SlrR-SinR) and gene transcription (SinR $s(r R)$. The loop is self-reinforcing in that inhibition (titration) of SinR by SlrR derepresses slrR, resulting in the accumulation of SlrR, which in turn further inhibits (titrates) SinR. The SinR SlrR switch has an SlrR LOW state (left loop) in which autolysin and motility genes are ON, and an SlrR HIGH state (right loop) in which these genes are OFF. The switch can be driven into the SlrR HIGH state either stochastically by noise during growth or deterministically during biofilm formation (see the text). Transcriptional regulation is indicated in red, and protein-protein interactions are indicated in blue. to the slrR promoter (and other SinR targets). Thus, the binding of SlrR to SinR causes derepression of its own gene, thereby creating a self-reinforcing loop. At the same time, the SinR•SlrR complex, but neither SinR alone nor SlrR alone, is a potent repressor of autolysin genes. Finally, evidence indicates that the double-negative feedback loop is an epigenetic switch that locks growing cells in alternative states of cell chaining and motility.

\section{Results}

\section{SlrR controls cell chaining during biofilm formation}

The starting point for this investigation was the question of how cell chaining is regulated during biofilm formation. Cells in the biofilm are predominantly in the form of long chains, which are bundled together by an extracellular matrix (Fig. 2A; Branda et al. 2001; Kearns et al. 2005; Kobayashi 2007). Such chains can be seen after growth in biofilm-inducing medium on agar, and in the pellicles that form at the air/liquid interface of standing cultures (Supplemental Fig. S1). We found that a mutant of $\operatorname{slr} R$ (YC131) is defective in cell chaining under both conditions (Fig. 2A). Furthermore, the effect of the slrR mutation was complemented by providing a copy of $s l r R$ (under the control of its native promoter) at the amyE locus on the chromosome (data not shown). Kobayashi (2007) has independently observed that an slrR mutant is blocked in chaining.

To investigate these observations further, we introduced into an $\operatorname{slr} R$ mutant a construct that contained an IPTG-inducible copy of $\operatorname{slr} R$ (slrR under control of the hyspank promoter $\mathrm{P}_{h y}$-slrR). The results of Figure 2B show that cell chaining occurred in an IPTG-inducible manner in the engineered strain. That the chains (and other chains observed in this study) were composed of linked cells that had undergone septation but had not separated from each other was confirmed by staining with the vital membrane stain FM4-64 (data not shown). SlrR is known to stimulate the production of the matrix protein TasA (Chu et al. 2008; Murray et al. 2009). We wondered whether the effect of slrR induction on cell chaining was indirectly due to increased TasA production, which we investigated using the $\mathrm{P}_{h y}$-slrR construct. The results of Figure $2 \mathrm{C}$ show that induction of chaining by the addition of IPTG was not dependent on TasA. It was also not dependent on eps, as judged by use of a mutation $(\Delta e p s H)$ in the eps operon (Fig. 2D). Therefore, cell chaining is not dependent on extracellular matrix production, and SlrR evidently has two distinct roles: stimulating matrix production, and promoting chaining.

\section{SlrR represses autolysin genes}

The separation of daughter cells after division is governed by peptidolglycan-degrading enzymes called autolysins (Blackman et al. 1998; Smith et al. 2000; Vollmer et al. 2008). How does SlrR control cell chaining? The simplest hypothesis is that SlrR is a repressor of the autolysin genes. Two autolysin genes that are known to play a key role in cell separation during vegetative growth are lytC 
Chai et al.

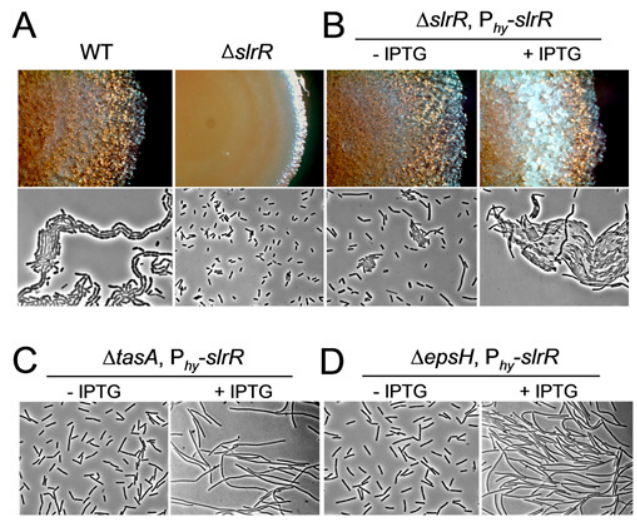

Figure 2. SlrR controls cell chaining during biofilm formation. (A) Cells from a colony of wild-type strain (WT) grown on solid biofilm medium exhibited extensive chaining, whereas $\Delta s \operatorname{lr} R$ mutant cells (strain YC131) were impaired in chaining. $(B)$ Induction of slrR from an IPTG-inducible promoter $\left(\mathrm{P}_{h y}-s / r R\right.$; strain YC280) caused extensive cell chaining in cells grown on solid biofilm medium. (The more wrinkled appearance of the $\Delta$ slrR mutant in $B$ than in $A$ is due to leakiness of $\mathrm{P}_{h y}-s \operatorname{lr} R$.) $(C, D)$ Neither $\Delta$ tas $A$ (strain YC283; C) nor $\Delta$ epsH (strain YC282; $D$ ) was able to block cell chaining induced by overproduction of SlrR.

and lytF (Ohnishi et al. 1999; Chen et al. 2009). The lytC and $l y t F$ genes are transcribed under the direction of the alternative $\sigma$ factor $\sigma^{\mathrm{D}}$, which also directs the transcription of genes for motility and chemotaxis (Margot et al. 1999; Chen et al. 2009). To test the hypothesis that SlrR represses these $\sigma^{\mathrm{D}}$-dependent genes, we fused the promoters for the lyt $A B C$ operon, which contains $l y t C$, and lytF to the lac Z gene, creating $\mathrm{P}_{\text {lytA- }}-1 a c Z$ and $\mathrm{P}_{\text {lyt }}-1 a c Z$, respectively. We then introduced the fusions into the wild type and into strains mutant for $\operatorname{slr} R(\Delta \operatorname{slr} R)$, or the $\sigma^{\mathrm{D}}$-encoding gene $\operatorname{sig} D(\Delta \operatorname{sig} D)$, or both $\operatorname{sir} R$ and $\operatorname{sig} D$ $(\Delta \operatorname{slr} R \Delta \operatorname{sig} D)$. The results shown in Figure 3, $\mathrm{A}\left(\mathrm{P}_{\text {lyta-lacZ }}\right)$ and $\mathrm{B}\left(\mathrm{P}_{\text {lytF- }}-\mathrm{lacZ}\right)$, demonstrate that expression of both fusions was somewhat higher in the slrR mutant than in the wild type $\left(\sim 120 \%\right.$ and $\sim 35 \%$ higher for $\mathrm{P}_{\text {lytA }}$-lacZ and $\mathrm{P}_{\text {lytF }}$-lac $Z$, respectively) in the early stationary phase in biofilm-inducing medium. The results also show that expression of lytC and lyt $F$ indeed depended on $\sigma^{\mathrm{D}}$, because both fusions showed little activity in the absence of the alternative $\sigma$ factor (Fig. 3A,B). The above results are consistent with the idea that SlrR negatively regulates lyt $C$ and lytF. However, the effect of the absence of SlrR seemed too small to account for the strong effect of the mutation on cell chaining (Fig. 2A).

A possible explanation comes from the fact that $s / r R$ is under the direct negative control of SinR (Chu et al. 2008; Kobayashi 2008). SinR-controlled genes, such as the eps and yqxM operons, are expressed in a bimodal pattern in shaking culture, with a small number of $\mathrm{ON}$ and a large number of OFF cells in the population (Chai et al. 2008; Vlamakis et al. 2008). We wondered whether expression of $\operatorname{slr} R$ also occurs in only a subpopulation of cells. If so, this bimodality could explain why only a mild effect was seen on lyt $C$ and lytF expression when slrR was mutant, as we were measuring $\beta$-galactosidase synthesis averaged among all cells in the population. To investigate whether $s l r R$ was $\mathrm{ON}$ in only a subpopulation of cells, we constructed a fusion of the promoter for slrR to the gene for the green fluorescent protein (GFP), creating $\mathrm{P}_{s I r R^{-}} g f p$. We integrated the fusion into the chromosome at the amyE locus and examined $\mathrm{P}_{\text {slrR }}{ }^{-g f p}$ expression by fluorescence microscopy. As shown in Figure 3C, only a small subpopulation of cells actively expressed $\mathrm{P}_{s l r R}-g f p$. Indeed, this subpopulation corresponded to cells that were in the form of chains. We infer that SlrR does repress lyt genes strongly, but only in the subpopulation of cells in which $\operatorname{slr} R$ is expressed at high levels. If so, then we should see strong repression of lyt gene expression using $\mathrm{P}_{h y}-s l r R$, which should lead to slrR expression in all cells. Indeed, when this construct was used, overall expression of $\mathrm{P}_{\text {lytF- }}$-lac $Z$ was markedly repressed in the presence of IPTG during early stationary phase under shaking culture conditions (Fig. 3D).

\section{Cell chaining depends on both SlrR and SinR}

The slrR gene lies downstream from $\sin I$ and $\sin R$ in the regulatory circuit that controls both matrix production and chaining (Fig. 1; Chu et al. 2008). We predicted that $\sin I$ and $\sin R$ should also have strong effects on cell chaining. In fact, and as shown in Figure 4A, a null mutation in $\operatorname{sinI}$, which greatly decreased $s l r R$ expression (Chu et al. 2008), caused an almost complete loss in cell

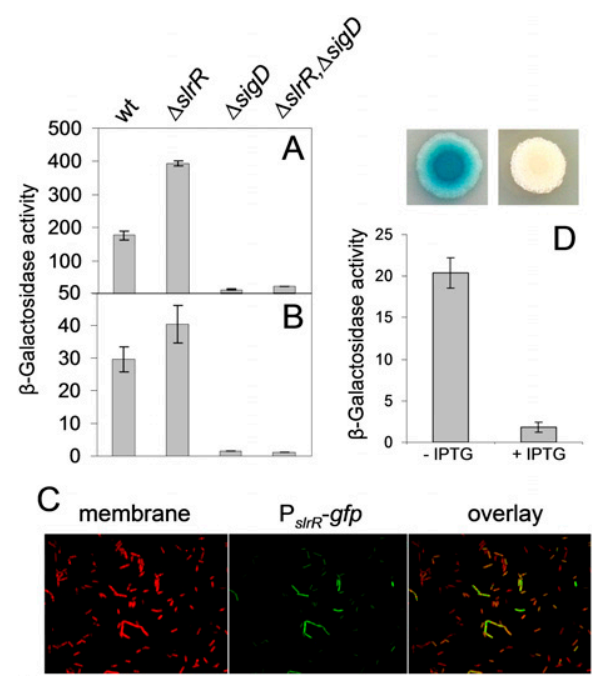

Figure 3. SlrR represses autolysin genes. $(A, B)$ Expression of $\mathrm{P}_{\text {lyt } A}$-lac $Z(A)$ or $\mathrm{P}_{\text {lyt }}$-lacZ $(B)$ in wild-type cells and in cells mutant for $\operatorname{slr} R, \operatorname{sig} D$, or both $\operatorname{slr} R$ and $\operatorname{sig} D$. Cells were grown in shaking culture in biofilm medium. $(C)$ Fluorescence microscopy of wild-type cells harboring a $\mathrm{P}_{\text {slrR }}-g f p$ fusion (YC175) grown in shaking culture in biofilm medium. The left panel shows cells stained in red with the membrane dye FM4-64, the middle panel shows fluorescence (green) from GFP, and the right panel is an overlay. (D) Expression of $\mathrm{P}_{\text {lytF }}-1 a c Z$ in a strain (YC600) that contains an IPTG-inducible copy of slrR grown on solid (top) or in liquid (bottom) biofilm medium in the absence or presence of an inducer. 


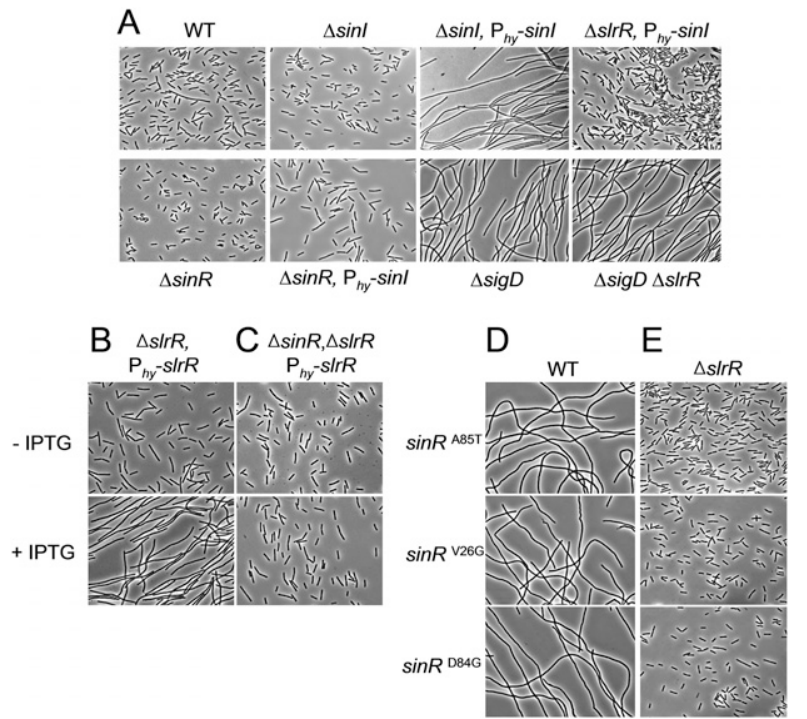

Figure 4. Chaining phenotype of mutants grown in shaking culture in biofilm medium. (A, top row) A $\Delta \sin I$ mutant (RL3853) was impaired in chaining, overexpression of $\operatorname{sinI}$ (YC227; a functional gfp-sinI translational fusion was used) promoted extensive cell chaining, and a $\Delta s l r R$ mutation reversed the chaining phenotype caused by overexpression of $\sin I$ (YC589). (Bottom row) A $\Delta$ sinR mutant (RL3856) showed little chaining (the strain contained an $\Delta$ eps $H$ mutation to prevent aggregation), a $\Delta \sin R$ mutation reversed the chaining phenotype caused by overexpression of $\sin I$ (YC228), a $\Delta \operatorname{sig} D$ mutant showed extensive cell chaining (RL4169), and the chaining phenotype was not reversed by $\Delta$ slrR (YC205). (B,C) Induction of slrR (from $\mathrm{P}_{h y}$-slrR) promoted chaining in shaking culture (strain $\mathrm{YC} 280 ; B$ ) but not in a strain that with a $\Delta \sin R$ mutation (strain YC284;C). $(D, E)$ Shown are point mutants of $\sin R$ that promoted chaining (D shows strains YC606, YC607, and YC608, respectively, from top to bottom panels). (E) Chaining caused by the point mutations was reversed by the presence of a $\Delta s \operatorname{lr} R$ mutation (strains YC620, YC621, and YC622, respectively, from top to bottom).

chaining in shaking culture in biofilm-inducing medium. (Note that, under shaking culture conditions in biofilminducing medium, only a modest proportion of wild-type cells form chains [Fig. 4A] as comapred with cells embedded in biofilms in which a high proportion of cells are in chains [Fig. 2A].) On the other hand, overexpression of $\operatorname{sinI}$ from an IPTG-inducible promoter caused extensive cell chaining throughout the cell population (Fig. 4A). Furthermore, this extensive chaining phenotype was almost completely reversed by introducing an slrR mutation into that strain (Fig. 4A), confirming that slrR lies downstream from sinI in the pathway controlling cell chaining.

Because SinR is a repressor of $\operatorname{slr} R$, and because $\operatorname{slr} R$ expression is derepressed in a $\sin R$-null mutant (Chu et al. 2008), we might have expected that a $\sin R$-null mutation would (like sinI overexpression) promote a high level of chaining. However, as observed previously (Kearns et al. 2005), a null mutant of $\sin R$ exhibited the opposite phenotype: little cell chaining (Fig. 4A). Furthermore, a $\Delta \sin R$ mutation reversed the extensive cell chaining phenotype caused by overexpression of $\sin I$ (Fig. 4A).

The above experiments were performed using cells harboring a mutation in the eps operon $(\Delta e p s H)$ to prevent cell aggregation. The $\Delta e p s H$ mutation itself had no measurable effect on cell chaining (Fig. 2D). In cells that are wild type for eps, the absence of SinR causes extensive aggregation (Kearns et al. 2005). In these aggregates, the cells are bundled tightly together but are separated from each other along their long axis and do not form chains (Kearns et al. 2005). In toto, these findings indicate that both SinR and SlrR are required for cell chaining.

That chaining depends on both SinR and SlrR is further indicated by the following experiment. As we showed, overexpression of $s$ lr $R$ from an IPTG-inducible promoter dramatically enhanced cell chaining during biofilm formation (Fig. 2B). This was also seen when cells were grown in shaking culture conditions (Fig. 4B). However, when SlrR was overproduced in a $\sin R$-null mutant strain, little cell chaining was seen (Fig. 4C).

\section{Certain point mutations of sinR promote cell chaining}

We showed that a null mutant of $\sin R$ is impaired in cell chaining (Fig. 4A). Historically, however, the sinR gene was called flaD (for flagellaless), because the original mutants exhibited a flagellaless and filamentous phenotype (Pooley and Karamata 1984). These filamentous (or hyperchaining) mutants were mapped to $\sin R$ (flaD1 and flaD2) and were found to be point mutations that caused single-aminoacid substitutions in SinR (Margot et al. 1996). We infer that the flaD1 and flaD2 alleles impart the opposite phenotype (hyperchaining) to that of the null mutation (little chaining).

To investigate the hypothesis that certain singleamino-acid substitutions in SinR can cause a hyperchaining phenotype, we devised a screen for mutant alleles of $\sin R$ that promote increased chaining (see the Materials and Methods). We recovered eight different single-aminoacid substitution mutants of SinR that caused conspicuous hyperchaining phenotypes (Fig. 5). Two substitutions were located in the DNA-binding domain of SinR (V26 and A27), and six (D55, E67, E79, D84, and A85; two different substitutions were recovered at D84) were clustered in the domain believed to be responsible for SinR-SinR interactions (Fig. 5; Lewis et al. 1996).

We picked three mutants for further study: V26G, a substitution in the DNA-binding domain; A85T, a substitution in the SinR-SinR interaction domain; and D84G, another substitution in the SinR-SinR domain that corresponds to the classic flaD1 mutation (Margot et al. 1996). All three mutants exhibited extensive chaining when grown in biofilm-inducing medium under shaking culture conditions (Fig. 4D). Also, and as expected, all three mutations caused overexpression of $\operatorname{sir} R$ as well as of matrix genes (Fig. 5; Supplemental Fig. S2).

Finally-and to characterize further the cell chaining function of the V26G, A85T, and D84G mutant proteins-we introduced an $s / r R$-null mutation $(\Delta s \operatorname{lr} R)$ into cells harboring the three mutant alleles of $\sin R$. 
Chai et al.

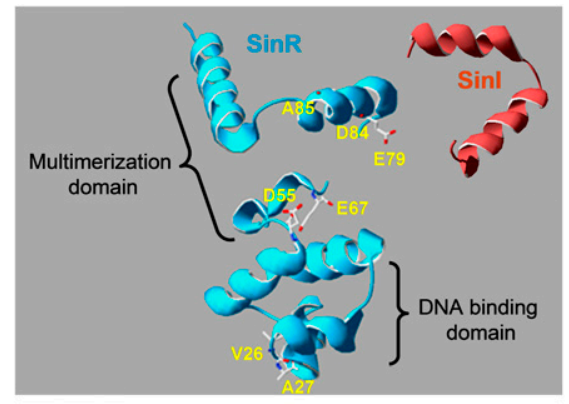

\begin{tabular}{|c|c|c|c|}
\hline Strain & mutation in $\sin R$ & matrix production & cell chaining \\
\hline YC581 & WT & + & + \\
\hline YCS1 & E67Y & +++ & +++ \\
\hline YCS2 & D55Y & +++ & +++ \\
\hline YCS4 & D84N & +++ & +++ \\
\hline YCS5 & A27T & +++ & +++ \\
\hline YCS6 & A85T & +++ & +++ \\
\hline YCS7 & V26G & +++ & +++ \\
\hline YCS11 & E79K & +++ & +++ \\
\hline YCS12 & D84G & t++ & +++ \\
\hline
\end{tabular}

Figure 5. Amino acid substitutions in SinR that promote chaining are clustered in two domains. Two residues (V26 and A27) are located in the DNA-binding domain, whereas the other five (D55, E67, E79, D84, and A85) are clustered in the multimerization domain that is responsible for SinR-SinR interactions.

Figure 4E shows that the resulting, doubly mutant cells had lost the robust chaining phenotype of the parent strains (Fig. 4D). We conclude that, like wild-type SinR, the capacity of the mutant SinR proteins to promote chaining depends on SlrR.

Both the SinR-SinR interaction and the DNA-binding domains of SinR are believed to be needed for SinR to adhere to its operators (Lewis et al. 1996, 1998; Kearns et al. 2005). Thus, a simple explanation for the classic filamentous phenotype of certain $\sin R$ alleles is that the amino acid substitution mutant proteins are impaired in SinR-SinR interaction or in recognition of the slrR promoter and are therefore defective in repressing slrR (which results in overexpression of slrR), but are not defective in promoting cell chaining. In other words, we hypothesize that SinR has two functions: repression of slrR (and the yqxM and eps operons), and a separate function that promotes cell chaining, presumably by contributing to the repression of autolysin genes. Evidence presented below indicates that this second function is to bind to SlrR to create a heteromeric complex that represses autolysin genes.

\section{SlrR interacts with SinR}

In toto, the results so far indicate that both SlrR and SinR are required for chaining. How are we to explain this requirement for both proteins? We speculated (1) that SlrR and SinR bind to each other to form a heteromeric complex, and (2) that such a complex (but neither SlrR nor SinR alone) is needed for repressing autolysin genes. In this section, we investigate whether the proteins bind to each other, and in the next two sections, we investigate whether the hypothesized complex binds to the promoter region of autolysin genes.
To investigate whether SlrR and SinR form a heteromeric complex, we carried out the following pull-down experiments. We mixed a lysate from Escherichia coli cells that were engineered to produce a His $_{6}-$ SinR fusion protein with a lysate of a $B$. subtilis strain that was deleted for $\sin R$ and hence overproduced SlrR. We applied the mixture to Ni-NTA agarose beads (New England Biolabs). Using antibodies that cross-react with both SinR and SlrR (Chai et al. 2009), we detected $\mathrm{His}_{6}$-SinR as well as SlrR in the eluate from the beads (Fig. 6A). Indeed, SlrR was retained almost quantitatively on the beads, with relatively little present in the flow-through. In a control experiment with an E. coli lysate from cells lacking His $_{6}{ }^{-}$ SinR, little or no SlrR was found in the eluate from the beads (Fig. 6A). These results indicate that SlrR forms a complex with SinR.

\section{A heteromeric complex of SlrR and SinR binds to the promoters for autolysin genes}

To investigate whether the SlrR $\bullet \operatorname{SinR}$ complex binds to the promoter region of autolysin genes, we carried out electrophoretic mobility shift assays (EMSAs). We initially used a radiolabeled DNA probe that contained the promoter region for the lyt $A B C$ operon. The EMSA experiments were carried out with purified SinR and a purified SlrR fusion protein, glutathione S-transferase (GST)-SlrR, in which SlrR was joined to GST. First, we tested SinR alone, which was unable to cause a mobility shift, even at the highest concentration of protein used (1000 nM) (Fig. 6B). Next, we tested GST-SlrR alone, which did cause the appearance of two species with retarded mobility (Fig. 6C). However, even at the highest concentration of protein $(1000 \mathrm{nM})$ not all of the probe was shifted (Fig. 6C, top panel, right lane).

We then investigated whether SinR would stimulate the ability of GST-SlrR to bind to the promoter sequence. To do this, we carried out the EMSA with increasing amounts of GST-SlrR, but this time in the presence of a fixed amount of SinR (Fig. 6D). The results show that the presence of $\operatorname{SinR}(500 \mathrm{nM})$, which by itself had not shifted the DNA probe (Fig. 6B, top panel, fifth lane), greatly stimulated the DNA-binding capacity of GSTSlrR; almost all of the DNA probe was shifted, even at the very lowest concentration of GST-SlrR (63 nM) (Fig. 6D).

Conversely, we carried out a gel shift experiment in which we added increasing amounts of SinR in the presence of a fixed but low amount of GST-SlrR $(63$ $\mathrm{nM}$ ), which by itself had shifted only a small amount of the DNA probe (Fig. 6C, top panel, second lane). As shown in Figure 6E, increasing amounts of SinR caused a substantial proportion of the probe to be shifted when the low amount of GST-SlrR was used (Fig. 6E). In other words, much more of the probe was shifted by the low amount of GST-SlrR when increasing amounts of SinR were added.

Similar results to those described above were obtained when we used as a probe DNA containing the lytF promoter (Supplemental Fig. S3A). Finally, and as a negative control, we used as a probe DNA that contained the 

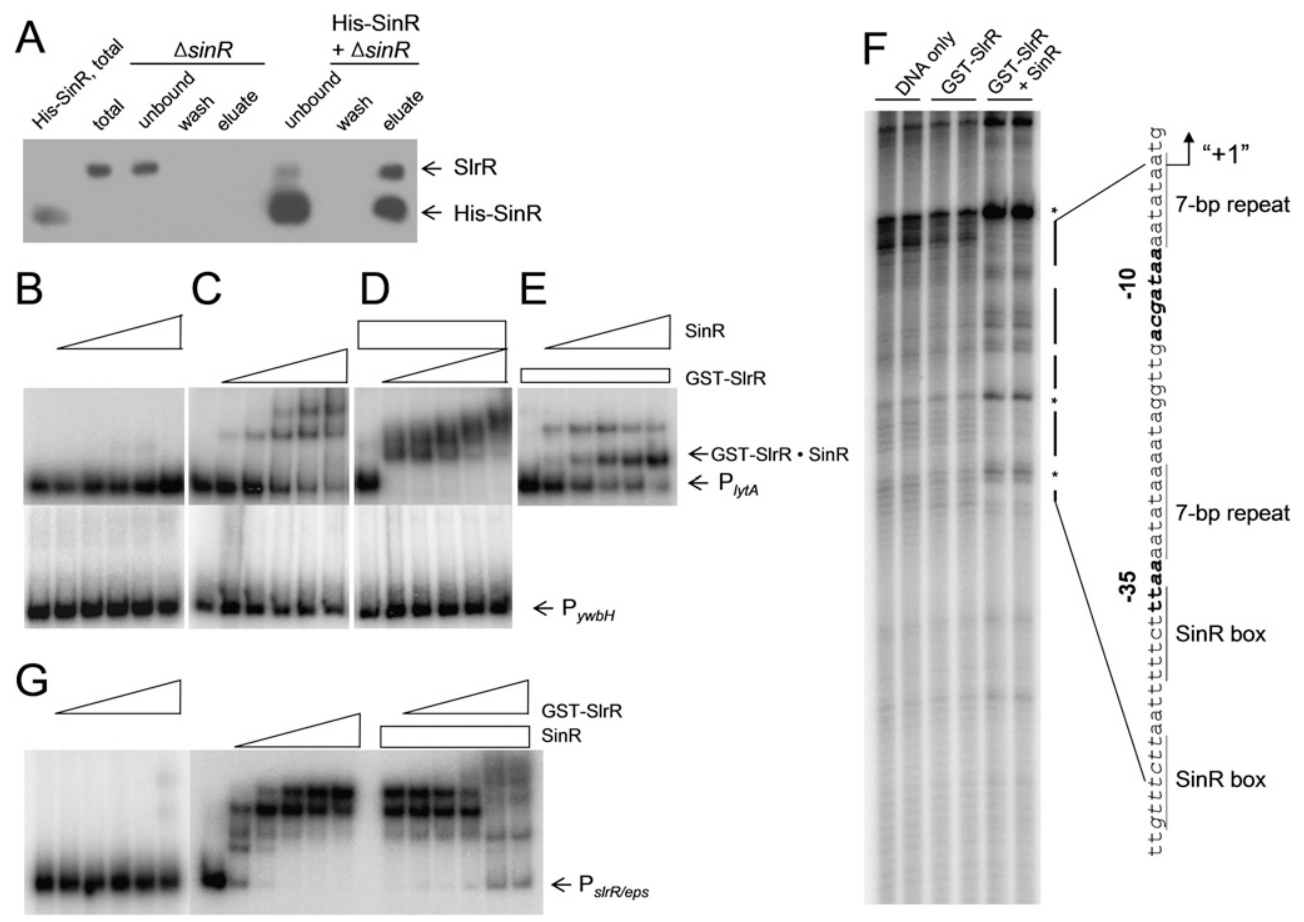

Figure 6. SlrR and SinR form a complex that binds to the promoter for the lytABC operon but not the control region for slrR and eps. (A) Shown is a pull-down experiment in which both $\mathrm{His}_{6}-\mathrm{SinR}$ and SlrR were detected in the eluate from Ni-NTA beads using antibodies that cross-reacted with both proteins. In a control without His 6 -SinR, SlrR was detected only in the flow-through fraction (unbound). $(B-E)$ The top panels show EMSAs using a radiolabeled DNA probe that contained the promoter for the lyt $A B C$ operon $\left(\mathrm{P}_{\text {lyt } A}\right)$, purified GST-SlrR fusion protein, and SinR. In $B$ and $C$, increasing SinR $(B)$ or GST-SlrR $(C)$ was added at $0,62.5,125,250,500$, and $1000 \mathrm{nM}$. In $D$, a fixed amount of $\operatorname{SinR}(500 \mathrm{nM})$ was mixed with increasing GST-SlrR at 0, 62.5, 125, 250, 500, and 1000 nM. In $E$, a fixed amount of GST-SlrR $(63 \mathrm{nM})$ was mixed with increasing SinR at 0,62.5, 125, 250, 500, and $1000 \mathrm{nM}$. The bottom panels in $B-D$ are control experiments in which the proteins were added at the same concentrations as in the top panels except that the radiolabeled DNA probe contained the promoter for an unrelated $y w b H$ gene. $(F)$ DNase I footprinting experiment to map the binding site for heteromeric SlrR $\bullet$ SinR in the promoter region for the lyt $A B C$ operon. In the middle two lanes, $1 \mu M$ GST-SlrR alone was incubated with a DNA probe, whereas in the right two lanes, $1 \mu \mathrm{M}$ both GST-SlrR and SinR were added. The indicated region of $\sim 47$ bp was protected from DNase I in the presence of both GST-SlrR and SinR. Bands that were protected from DNase I or were hypersensitive to the nuclease are indicated by vertical bars and asterisks, respectively. Within the protected region, two SinR operator-like sequences (indicated as SinR box) and two identical repeats are labeled. Also labeled are the -35 and -10 regions and the +1 start site for the $\sigma^{\mathrm{D}}$-dependent promoter. $(G)$ EMSAs using a radiolabeled DNA probe for the intergenic control region for slrR and eps $\left(\mathrm{P}_{\text {slrR/eps }}\right)$, and purified GST-SlrR fusion protein and SinR. In the left panel, increasing amounts of GST-SlrR were added $(0,31.2,62.5,125,250$, and 500 nM). In the middle panel, increasing amounts of $\operatorname{SinR}$ were added $(0,15.6,31.2,62.5,125$, and $250 \mathrm{nM})$. In the right panel, a fixed amount of $\operatorname{SinR}(125 \mathrm{nM})$ was mixed with increasing amounts of GST-SlrR (0, 31.2, 62.5, 125, 250, $500 \mathrm{nM})$.

promoter region of an unrelated gene, ywbH. Neither SlrR nor SinR alone, nor a mixture of two proteins, was able to shift the control DNA at all of the concentrations tested (Figs. 6B-D, bottom panels). We conclude that SlrR binds specifically to the promoter regions for the two autolysin genes tested, and that this binding is substantially stimulated by the presence of SinR. In toto, the results are consistent with the idea that the repressor for lyt $A B C$ and lyt $F$ is an SlrR•SinR heteromeric complex.

\section{An $\operatorname{SlrR} \bullet \operatorname{Sin} R$ heteromeric complex covers the promoter for lytABC.}

Next, we carried out a DNase I footprinting experiment to map the binding site for the SlrR •SinR complex in the lyt $A B C$ promoter region. When equal molar concentrations of GST-SlrR and SinR were incubated with the DNA probe, we observed bands that were protected from DNase I and bands that were hypersensitive to the nuclease over a large region ( $\sim 77$ base pairs [bp]). These bands extended from about the transcription start site to $\sim 46$ bp upstream of the start site (indicated by bars and asterisks in Fig. 6F). Thus, the complex almost entirely covered the promoter for lyt $A B C$. In comparison, no significant protection was observed when footprinting was carried out with GST-SlrR alone (Fig. 6F, middle two lanes) or SinR alone (data not shown).

Within the region protected by GST-SlrR and SinR, we observed two putative SinR operators: one with a singlebase-pair mismatch to the 7-bp consensus sequence "GTTCTTT" (Chu et al. 2006), and the other with a 2-bp mismatch. We also observed two identical 7-bp repeat sequences: "AATATAA" (underlined in Fig. 6F). The simplest (but provisional) interpretation of these 
Chai et al.

results is that two molecules of SlrR bind to the repeated AATATAA sequences, and two molecules of SinR bind to the two SinR-like binding sequences.

\section{SlrR inhibits the binding of SinR to the control regions for slrR, eps, and yqxM}

We showed that, by binding to SinR, the SlrR protein confers a new property on SinR; namely, the capacity to bind to and repress autolysin genes. We wondered whether the binding of SlrR to SinR might also alter the affinity of SinR for the operators that it otherwise binds to on its own; namely, those for slrR, eps, and yqxM. To investigate this, we carried out EMSAs using as a probe DNA containing the $\sim 200$-bp intergenic region between eps and slrR. The eps operon and the slrR gene are divergently transcribed from a common control region that contains multiple SinR-binding sites (Kearns et al. 2005). Increasing amounts of GST-SlrR (from 0 to $500 \mathrm{nM}$ ) was not able to shift the DNA probe (Fig. 6G, left panel), whereas increasing amounts of SinR (from 0 to $250 \mathrm{nM}$ ) by itself were highly effective in retarding the mobility of a DNA probe that contained the control region for slrR and eps, causing the formation of higher-order, slowmigrating species (Fig. 6G, middle panel). However, when increasing amounts of GST-SlrR (from 0 to $500 \mathrm{nM}$ ) were added to a fixed amount of $\operatorname{SinR}(125 \mathrm{nM})$ that by itself was able to fully cause the formation of the higher-order complex, the gel shift was reversed with the partial reappearance of a free probe and the elimination of the slow-migrating species (Fig. 6G, right panel, right two lanes). Thus, SlrR inhibits the capacity of SinR to bind to the control region for its own gene (slrR) as well as for eps. We wondered whether SlrR would likewise antagonize the binding of SinR to the $y q x M$ promoter. Indeed, and as shown in Supplemental Figure S4A, the addition of SlrR reversed the binding of SinR to DNA containing the yqxM promoter (Supplemental Fig. S3C).

The above results suggest that SinR, SlrR, and $\operatorname{sir} R$ are components of a self-reinforcing, double-negative feedback loop in which SlrR antagonizes (titrates) SinR, thereby stimulating derepression of slrR. Derepression would result in yet more SlrR production, thereby further enhancing derepression of slrR as well as the matrix operons eps and yqxM. Indeed, and in support of this hypothesis, we found that, in a strain that contains both a $P_{\text {slrR }}$-lac $Z$ fusion and an IPTG-inducible copy of $s l r R$, the activity of $\mathrm{P}_{\text {slrR }}-\mathrm{lac} Z$ was higher in the presence of the inducer than in its absence (Supplemental Fig. S4). These findings also explain the previous observation that overexpression of $s$ lrR increases the expression of eps and yqxM (Kobayashi 2008; Murray et al. 2009).

As reported prevously, biofilm formation is triggered by the production of the anti-repressor SinI, which inhibits SinR (Kearns et al. 2005; Chai et al. 2008). We now propose that SinR is subject to anti-repression by the successive action of SinI and then SlrR. In other words, SinI sets in motion a chain of events that leads to the appearance of an additional anti-repressor, SlrR, which augments the action of SinI.
SinR and SlrR control cell chaining during exponential phase growth

Cells in the mid-exponential phase of growth are a mixture of two cell types: motile cells, and chains of sessile cells (Kearns and Losick 2005). We wondered whether the $\mathrm{SlrR} \bullet \operatorname{SinR}$ complex is also responsible for chaining under these conditions. We therefore examined the chaining phenotype of growing cells during exponential phase for the wild type and for $\Delta \sin I, \Delta \sin R$, and $\Delta \operatorname{sir} R$ mutants. We performed the analysis in two genetic backgrounds: that of 3610, an undomesticated strain used for biofilm studies, and that of PY79, a laboratory strain. 3610 exhibits a high level of $\sigma^{\mathrm{D}}$ activity and is biased toward motile cells, whereas PY79 (due to a mutation in $s w r A$ ) exhibits lower $\sigma^{\mathrm{D}}$ activity and produces a relatively high proportion of chains (Kearns and Losick 2005). We also introduced a $\mathrm{P}_{\text {hag }}$-gfp fusion (green in Fig. 7) into the strains as a reporter for $\sigma^{\mathrm{D}}$-directed transcription. The cells were stained with the dye FM4-64 to visualize membranes (red in Fig. 7).

As reported previously (Kearns and Losick 2005), both wild-type strains exhibited a bimodal pattern of cell types: single cells that were $\mathrm{ON}$ for $\mathrm{P}_{h a g}-g f p$, and chains of cells that were OFF (Fig. 7). Also, as expected, the proportion of chains was higher for PY79 (wild type in Fig. 7A) than for 3610 (wild type in Fig. 7B). Importantly, the proportion of cell chains was markedly reduced or completely absent in all three mutants in both the 3610 and PY79 backgrounds (Fig. 7). Thus, SinR and SlrR control cell chaining during exponential-phase growth as well as during biofilm formation.

We draw two inferences from these results. First, lyt $A B C$ and lytF are not the only targets of the SlrR $\bullet \operatorname{SinR}$

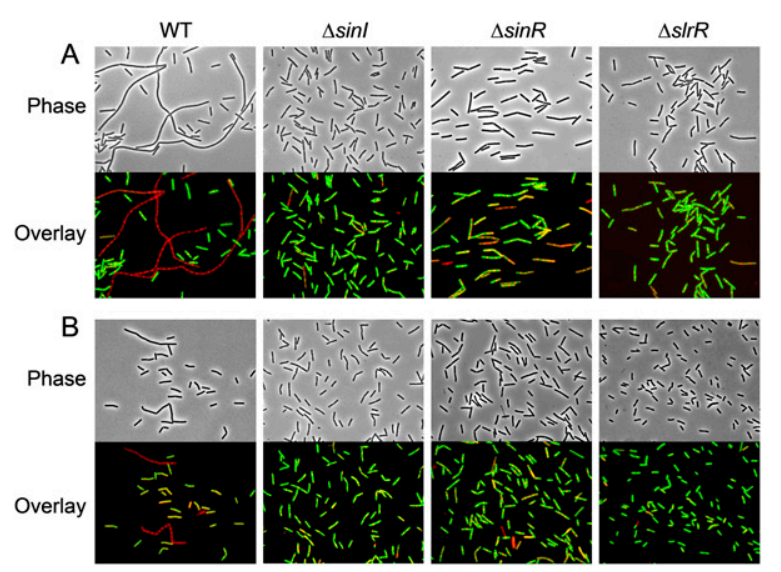

Figure 7. The SinR SlrR slrR switch controls cell chaining during exponential-phase growth. Shown are a domesticated strain $(\mathrm{PY} 79 ; A)$ and an undomesticated strain $(3610 ; B)$ and derivatives of each mutant for $\sin I, \sin R$, or $\operatorname{sir} R$. The strains contained a $\mathrm{P}_{\text {hag }}$-gfp fusion to visualize $\sigma^{\mathrm{D}}$-directed transcription (green). The cells were grown to mid-exponential phase in LB medium and stained with the membrane dye FM4-64 (red). PY79 and 3610 showed a mixture of single cells that were ON for $\sigma^{\mathrm{D}}$, and chains of cells that were OFF for $\sigma^{\mathrm{D}}$. All of the mutants were impaired in chaining and were ON for $\sigma^{\mathrm{D}}$. 
repressor. The results of Figure 7 suggest that the $\sigma^{\mathrm{D}}$-controlled hag gene, which encodes flagellin (LaVallie and Stahl 1989), is also under the negative control of SlrR•SinR. In confirmation of this inference, hag gene expression was decreased drastically throughout the population in the cell chaining-promoting amino acid substitution mutants of SinR described above (data not shown), and EMSAs showed that the SlrR•SinR complex binds to the promoter region for hag (Supplemental Fig. S3B). In toto, these findings raise the possibility that the $\mathrm{SlrR} \cdot \mathrm{SinR}$ complex is a repressor of additional, and perhaps many, members of the $\sigma^{\mathrm{D}}$ regulon.

The second inference is that the bimodal distribution of $\sigma^{\mathrm{D}}$-directed transcription is governed by the SinR SlrR double-negative feedback loop (Fig. 1B). We propose that the loop similarly creates a bistable switch that is responsible for switching $\sigma^{\mathrm{D}}$-transcribed genes ON or OFF. The switch can be biased in one direction or the other by $\sigma^{\mathrm{D}}$ levels, as demonstrated by the effects of mutations of $s w r A$, a gene that stimulates transcription of the operon that encodes $\sigma^{\mathrm{D}}$ (Kearns and Losick 2005). Nonetheless, swrA itself is not the basis for bistability in $\sigma^{\mathrm{D}}$-directed transcription. Instead, we propose that the self-reinforcing cycle of SlrR synthesis contributes to, or is entirely responsible for, determining whether $\sigma^{\mathrm{D}}$-directed transcription of autolysin and motility genes is ON or OFF.

\section{The double-negative feedback loop exhibits hysteresis}

If the SinR SlrR loop is a bistable switch, then it should exhibit hysteresis (Veening et al. 2008); that is, once activated, the circuit should remain on for long periods of time in the absence of the original stimulus. To address this question, we introduced the IPTG-inducible slr $R$ construct $\left(\mathrm{P}_{h y}-\mathrm{Slr} R\right)$ into the amyE locus on the chromosome of strain 3610, creating YC281. As a control, we integrated $\mathrm{P}_{h y}-s l r R$ into a derivative of the 3610 mutant for slr $R$, creating strain YC280. Both strains were grown in LB medium to the midexponential phase of growth. We then switched on cell chaining by treating the cells with IPTG.
As shown in Figure 8, before induction, YC281 cells exhibited a bimodal distribution of single cells and chains, with a strong bias toward single cells (Fig. 8A [top],G [column a]). YC280 cells showed little chaining before induction due to the $\Delta s l r R$ mutation (Fig. 8A [bottom], $\mathrm{H}$ [column a]). After growth in the presence of the inducer for $60 \mathrm{~min}$, both strains showed greatly increased levels of chaining (Fig. 8B [top and bottom], $\mathrm{G}, \mathrm{H}$ [columns $b]$ ). The cells were then washed to remove IPTG and diluted into fresh LB medium at hourly intervals to maintain exponential-phase growth. At 120 and 180 min after washing (two fourfold dilutions into fresh medium), the YC281 (slr $\left.R^{+}\right)$cells showed even more extensive cell chaining than before washing (Fig. 8D,E [top], G [columns d,e]). Moreover, and with additional rounds of dilution and growth in fresh medium, a high level of chaining could still be seen for as long as $5 \mathrm{~h}$ after washing (Fig 8F [top], G [column f]), representing $>10$ generations of growth. In contrast, chaining by the control strain YC280 $(\Delta s l r R)$ was not sustained after the removal of the inducer, with the cells returning to the short chain or single-cell state they were in before treatment with the inducer (Fig. 8D-F [bottom], H [columns $d-f]$ ). These results indicate that, once activated by a pulse of SlrR synthesis, the double-negative feedback loop remains locked in a state in which cell separation (autolysin) genes remain OFF for multiple rounds of cell division; that is, the loop exhibits hysteresis.

\section{Discussion}

The principal contribution of this investigation is the discovery of an epigenetic switch that controls $\sigma^{\mathrm{D}}$-directed transcription of genes for cell separation and motility during growth (Fig. 1). The switch consists of the proteins SinR and SlrR and the gene for SlrR. Results with mutants of SinR and SlrR and with cells engineered to produce SlrR in response to an inducer indicate that the switch is both necessary and sufficient to explain the bistability
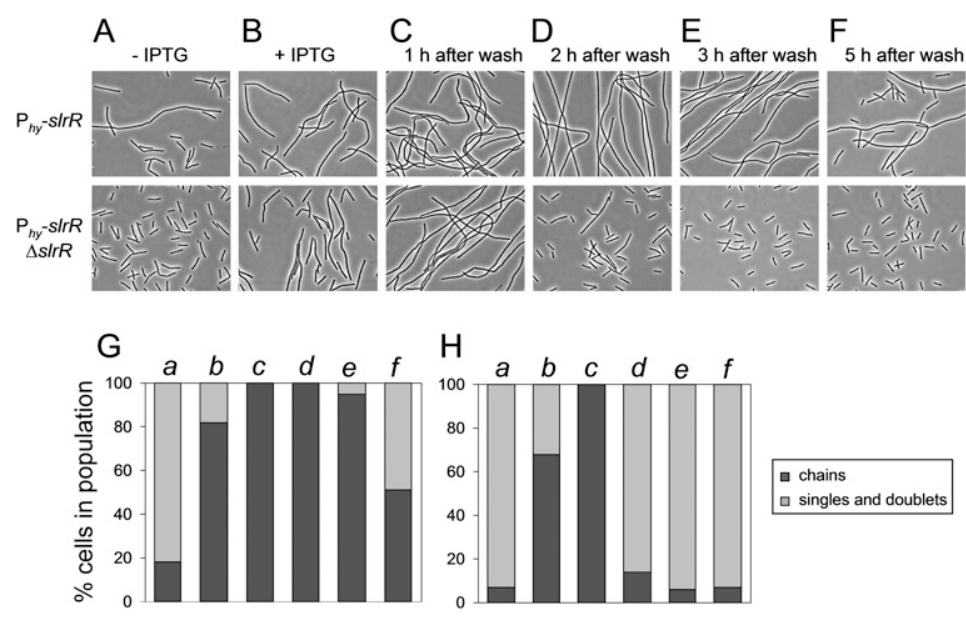

Figure 8. The feedback loop exhibits hysteresis. $(A$, top panel) Cells of an undomesticated $\left(s w r A^{+}\right)$strain bearing an IPTG-inducible copy of slrR (YC281) were largely in the form of single cells when grown in LB to mid-exponential phase in the absence of an inducer. (Bottom panel) A derivative of the above strain that was mutant for slrR (YC280) contained few chains under the same conditions. $(B)$ After treatment with $100 \mu \mathrm{M}$ IPTG for $60 \mathrm{~min}$ to induce SlrR production, the proportion of chains markedly increased for both strains. $(C-E)$ The cells were washed to remove IPTG, and samples were withdrawn and diluted fourfold into fresh LB medium at hourly intervals. At 120 and $180 \mathrm{~min}$ after suspension, cells of YC281 (top panels in $D, E$ ) were still largely in the form of chains, whereas cells of YC280 (bottom panels in $D, E)$ had largely reverted back to single cells. (F, top panel) After further rounds of growth and suspension in fresh LB medium, the YC281 cells were still largely in the form of chains. $(G, H)$ Quantitative results showing the ratio of chains versus single cells for YC281 $(G)$ and YC280 $(H)$ cells before and after induction shown in $A-F$. 
of cell separation and motility in growing cells. We propose that the SinR SlrR switch exists in two alternative stable states: a state in which SlrR levels are high (SlrR HIGH state), and a state in which SlrR levels are low (SlrR LOW state) (Fig. 1B). Autolysin and motility genes are OFF in the SlrR HIGH state and are ON in the SlrR LOW state.

Like the classic epigenetic switch of phage $\lambda$ in which the CI repressor represses the gene for Cro and the Cro repressor represses the gene for CI (Oppenheim et al. 2005), the SinR SlrR switch is a double-negative feedback loop. Unlike the $\lambda$ switch, however, the SinR SlrR switch involves protein-protein (SlrR-mediated inhibition of SinR) and protein-DNA (SinR-mediated repression of slrR) interactions. Also, like the well-studied ComK bistable switch, which governs entry into the state of genetic competence in B. subtilis (Maamar and Dubnau 2005; Maamar et al. 2007), the SinR SlrR switch is selfreinforcing in that SlrR inhibits the capacity of SinR to repress the gene for SlrR. (In effect, SlrR titrates SinR, thereby derepressing slrR.) The ComK switch is, however, a positive feedback loop in which ComK directly stimulates its own synthesis, as opposed to a double-negative loop in which one component (SlrR) indirectly stimulates its own synthesis (Maamar and Dubnau 2005). Finally, the SinR SlrR switch differs from these other examples of bistable switches in that it consists of three components (SinR, SlrR, and the SinR target slrR), whereas the phage $\lambda$ switch has four components (CI, Cro, and the DNAbinding sites for the two repressors) and ComK has two components (ComK and its binding site). A virtue of the three-component switch is that it not only creates bistability, but also repurposes one of the components: SinR.

During biofilm formation, SlrR synthesis is triggered by the synthesis of the anti-repressor SinI, which inhibits SinR, as we discuss below (Kearns et al. 2005; Chu et al. 2008). How, then, is SlrR synthesis triggered during growth in rich medium, when the levels of SinI are low? We propose that the switch is activated stochastically by noise in the expression of these same components. For example, under conditions of exponential-phase growth in which SpoOA P levels are low, sinI is largely silent in the cell population as a whole. Yet $\sin I$ must contribute to the switching because a sinI mutant is locked in the SlrR LOW (autolysin and motility genes ON) state in exponential-phase cells. We therefore hypothesize that stochastic and transient expression of $\sin I$ is a feature of exponentially growing cells. In only a subpopulation, enough SinI accumulates to cause derepression of $\operatorname{slr} R$, thereby triggering self-reinforcing synthesis of SlrR. This process could be aided by noise in the expression of $s / r R$ (that is, escape of $\operatorname{slr} R$ from SinR-mediated repression), which could push some cells to the brink of triggering self-reinforcing synthesis of SlrR. Triggered by stochastic events, self-reinforcing synthesis of SlrR would switch the SinR SlrR loop into the SlrR HIGH state (autolysin and motility genes OFF) and lock a subpopulation of cells in the form of chains (Fig. 1B).

We began this investigation by attempting to elucidate the mechanism that governs cell chaining during biofilm formation. We discovered, however, that the same circuitry that governs cell chaining in biofilms also controls cell chaining in growing cells, but with one important difference. During biofilm formation, the switch is not activated stochastically. Rather, it is driven into the SlrR HIGH state by the programmed synthesis of the antirepressor SinI (Fig. 1B); that is, in biofilm-inducing medium, the gene for the SinI anti-repressor is turned on by Spo0A $\sim$, a global regulator of post-exponential-phase gene expression (Fujita et al. 2005; Chai et al. 2008). Spo0A $\sim$ P-directed synthesis of SinI results in inhibition of SinR, and hence depression of the gene for SlrR (Fig. 1A). Thus, the activation of SpoOA $\sim \mathrm{P}$ in stationary phase sets in motion a cascade in which $\operatorname{Sin} R$ is successively inhibited by SinI and then by SlrR, resulting in derepression of SinR targets (i.e., slrR, eps, and yqxM) and repression of SlrR targets (i.e., lyt $A B C$ and lytF) through the formation of the SinR•SlrR complex. In other words, during biofilm formation, expression of $\operatorname{slr} R$ is not left to chance; it is a downstream consequence of the activation of SpoOA $\sim \mathrm{P}$.

Fascinatingly, SinI, SinR, SlrR, and SlrA are members of a family of homologous proteins that are linked to each other through intricate circuitry. We did not consider SlrA here because it does not contribute importantly to biofilm formation or cell chaining under laboratory conditions (unless it is artificially overexpressed) (Chai et al. 2009). Its synthesis is believed to be governed by an unknown environmental signal (Kobayashi 2008; Chai et al. 2009). Nonetheless, it is a paralog of SinI, and, like SinI, it is an anti-repressor for SinR (Chai et al. 2009). SlrR is itself a hybrid protein, with domains that are homologous to SinI and SlrA on the one hand, and with domains that are homologous to SinR on the other hand (Chu et al. 2008). In fact, then, $\operatorname{SinR}$ is subject to inhibition by three antirepressors: SinI, SlrA, and, as we observed, SlrR. Meanwhile, SinR, on its own, is a repressor of a particular set of promoters (slrR, eps, and yqxM), but can be repurposed by SlrR to bind to an entirely new set of promoters (lyt $A B C$, lytF, hag, and, perhaps, those for additional $\sigma^{\mathrm{D}}$-transcribed genes) by forming a SinR・SlrR heterocomplex.

In summary, a single, simple regulatory circuit is deployed by $B$. subtilis to control gene expression under different biological circumstances. Under conditions (stationary phase in poor medium) that promote the formation of architecturally complex communities, the SinR SlrR switch is part of a regulatory cascade that culminates in the formation of long chains of cells and in the production of an extracellular matrix that binds them together. Conversely, under conditions of exponential-phase growth in rich medium, the same circuit is an epigenetic switch that is activated stochastically to lock cells in alternative states of motility and cell chaining.

\section{Materials and methods}

General methods for media, strain construction, $\beta$-galactosidase asays, protein production, and purification

See the Supplemental Material for general methods for media, strain construction, $\beta$-galactosidase assays, protein production, and purification. 
Strains

See Supplemental Table S1 for a list of strains.

\section{Colony and pellicle formation}

For colony formation, cells were first grown to exponential growth phase in LB broth, and $3 \mu \mathrm{L}$ of these cultures were spotted onto solid MSgg medium containing 1.5\% Bacto agar. The plates were incubated at $23^{\circ} \mathrm{C}$. Images of the colonies were taken using a SPOT camera (Diagnostic Instruments). For pellicle formation, cells were grown to exponential phase, and $9 \mu \mathrm{L}$ of cultures were mixed with $9 \mathrm{~mL}$ of liquid medium in a sixwell microtiter plate (VWR). Plates were incubated for 2-3 d at $23^{\circ} \mathrm{C}$. Images of the pellicles were recorded similarly.

\section{Microscopy analysis}

For assays of cell chaining during pellicle formation, cells were cultured as described above. Cells were collected from pellicleforming wells after $48 \mathrm{~h}$ of incubation and were washed twice with cold PBS buffer. Cells were suspended in $50 \mu \mathrm{L}$ of cold PBS buffer and were analyzed using phase-contrast microscopy. For assays of cell chaining in shaking culture conditions, cells were collected in either early stationary phase when grown in MSgg medium, or mid-exponential growth phase when grown in LB medium at $37^{\circ} \mathrm{C}$. Cells were treated as described above for microscopy analysis. To induce expression of $s / r R$ from the hyspank promoter, $500 \mu \mathrm{M}$ IPTG (unless otherwise indicated) was added to cells grown to early exponential phase in broth medium, and the cells were allowed to continue to grow with shaking for one more hour at $37^{\circ} \mathrm{C}$ before being harvested.

For fluorescence microscopy analysis, cells were grown in either MSgg broth to early stationary phase or LB medium to mid-exponential phase. One milliliter of the culture was harvested. Cells were washed with cold PBS buffer twice and suspended in $50 \mu \mathrm{L}$ of PBS buffer. One microliter of diluted membrane-staining dye FM4-64 was mixed with suspended cells. Three microliters of FM4-64-treated cells were dropped to the center of an agar-coated microscopy slide. Cover slides were pretreated with polyl-lysine (Sigma). Samples were examined using an Olympus workstation BX61. Images were taken using an automated software program (SimplePCI) and analyzed with MetaMorph (Universal Imaging Corporation).

\section{Screen for chaining-promoting mutants of $\operatorname{Sin} R$}

B. subtilis cells mutant for ymcA are severely impaired for biofilm formation (Branda et al. 2004). We discovered previously that, after prolonged incubation, a $\triangle y m c A$ mutant was able to form pellicles due to the appearance of suppressor mutations (Kearns et al. 2005). Frequently, the suppressor mutations were mapped to $\sin R$. We also discovered that, often, those suppressors were rescued not only for matrix production, but also for cell chaining. A strain (YC581) was constructed as described above that contained a null mutation in $y m c A(\Delta y m c A)$ and a kanamycin resistance gene was inserted into a chromosomal locus immediately downstream from $\sin R$. The purpose of introducing the kan gene was to facilitate the transfer of suppressor mutations in $\sin R$ to another genetic background. The kan gene had no affect on biofilm formation and cell chaining (data not shown). After prolonged incubation of YC581 cells in standing MSgg medium, we were able to pick suppressors that formed pellicles. Among the suppressors obtained, 12 contained mutations in the $\sin R$ gene. Nine of the 12 suppressor mutations were found to rescue both matrix production and chaining, while the remaining three rescued matrix production, but not chaining, and they were not further characterized. All nine suppressor mutants contained point mutations that caused single-aminoacid substitutions in the SinR protein (Fig. 5; A85T substitution was obtained independently twice).

\section{Pull-down experiment}

The cleared lysate containing $\mathrm{His}_{6}$-SinR fusion proteins was prepared from the $E$. coli cells as described above, and was mixed in equal volume with cleared lysate from a B. subtilis $\Delta \sin R$ mutant (RL3856). Preparation of cleared lysate from B. subtilis RL3856 was as follows. Cells were inoculated into $100 \mathrm{~mL}$ of MSgg broth and grown at $37^{\circ} \mathrm{C}$ to early stationary phase. Cells were harvested and washed with $10 \mathrm{~mL}$ of cold phosphate buffer ( $20 \mathrm{mM}$ sodium phosphate, $200 \mathrm{mM} \mathrm{NaCl}, 10 \%$ glycerol, $1 \mathrm{mM}$ PMSF at pH 7.4). Cell pellets were suspended in $1 \mathrm{~mL}$ of cold phosphate buffer supplemented with $500 \mu \mathrm{g} \mathrm{mL}^{-1}$ of freshly made lysozyme solution and were incubated for $30 \mathrm{~min}$ on ice. Cells were further disrupted using sonication on ice. Cell lysate was centrifuged at $5000 \mathrm{rpm}$ for $5 \mathrm{~min}$ to remove cell debris, and were further centrifuged at $14,000 \mathrm{rpm}$ for $30 \mathrm{~min}$ at $4^{\circ} \mathrm{C}$. The mixture of the E. coli and the B. subtilis lysates was incubated for $30 \mathrm{~min}$ at $4^{\circ} \mathrm{C}$, applied to Ni-NTA agarose beads, and incubated for one more hour at $4^{\circ} \mathrm{C}$ with rotation. Proteins were eluted afterward, following the same procedures that were described above. Immunodetection of SinR and SlrR proteins was performed following a protocol that was published previously (Chai et al. 2009). The control experiment was performed similarly except that the E. coli lysate does not contain $\mathrm{His}_{6}$-SinR.

\section{EMSA}

The DNA probes for the promoter sequences of ly $A B C$, lyt $F$, and $\operatorname{slr} R$ were generated by PCR using chromosomal DNA of 3610 and primers $\mathrm{P}_{l y t A}-\mathrm{F} 1$ and $\mathrm{P}_{l y t A}-\mathrm{R} 1, \mathrm{P}_{l y t F}-\mathrm{F} 1$ and $\mathrm{P}_{l y t F}-\mathrm{R} 1$, and $\mathrm{P}_{\text {slrR }}-\mathrm{F} 1$ and $\mathrm{P}_{\text {slrR }}-\mathrm{R} 1$, respectively. See Supplemental Table $\mathrm{S} 2$ for a description of the primers. The DNA probes were digested with EcoRI, gel-purified, and filled in using klenow $\left(\right.$ exo $\left.0^{-}\right)$dTTP, and $\left[\alpha-{ }^{32} \mathrm{P}\right]$-dATP (New England Biolabs). EMSA was conducted following a protocol that had been described previously (Chai et al. 2009). When both SinR and GST-SlrR were applied in the assay, proteins were mixed and incubated for $15 \mathrm{~min}$ on ice first before the DNA probe was added.

\section{DNase I footprinting}

The DNA probe containing the promoter region for the lyt $A B C$ operon was amplified and end-labeled as described above. Radiolabeled DNA was mixed with purified SinR and/or GST-S1rR at indicated concentrations, and the mixture was incubated in $50 \mu \mathrm{L}$ of binding buffer $(20 \mathrm{mM}$ Tris buffer at $\mathrm{pH} 8.0,5 \mathrm{mM}$ $\mathrm{MgCl}_{2}, 0.1 \mathrm{mM}$ DTT, $50 \mu \mathrm{g} \mathrm{mL}^{-1} \mathrm{BSA}, 5 \mu \mathrm{g} \mathrm{mL}^{-1}$ poly-dI-dC, $10 \%$ glycerol) for $20 \mathrm{~min}$ on ice. The mixture was warmed up for $10 \mathrm{~min}$ at room temperature. To each mixture, $50 \mu \mathrm{L}$ of digestion mix - which contains $0.05 \mathrm{U}$ of DNase I (amplification grade, Invitrogen), $10 \mu \mathrm{L}$ of $10 \times$ DNase I digestion buffer, and $0.3 \mathrm{mM}$ DTT-was added and quickly mixed. The mixture was incubated for $30 \mathrm{sec}$ at room temperature. The reaction was stopped by addition of $5 \mu \mathrm{L}$ of $200 \mathrm{mM}$ EDTA. DNAs were precipitated by addition of $2.5 \mathrm{vol}$ of cold ethanol and $10 \%$ of $3 \mathrm{M}$ sodium acetate (pH 5.3), and were kept for $1 \mathrm{~h}$ at $-80^{\circ} \mathrm{C}$. DNAs were resuspended in $10 \mu \mathrm{L}$ of formamide loading buffer, and $5 \mu \mathrm{L}$ of the sample were loaded to $6 \%$ TBE sequencing gel (National Diagnostics). 


\section{Acknowledgments}

We are grateful to D. Kearns and J. Paulsson for helpful suggestions. We thank Drs. A. Camp and F. Chu for strains. This work was supported by NIH grants GM18569 to R.L. and GM58213 to R.K., and by grants from BASF to both R.L. and R.K. Y.C. was a fellow of the Jane Coffin Childs Foundation.

\section{References}

Bai U, Mandic-Mulec I, Smith I. 1993. SinI modulates the activity of SinR, a developmental switch protein of Bacillus subtilis, by protein-protein interaction. Genes \& Dev 7: 139148.

Blackman SA, Smith TJ, Foster SJ. 1998. The role of autolysins during vegetative growth of Bacillus subtilis 168. Microbiology 144: 73-82.

Bramhill D. 1997. Bacterial cell division. Annu Rev Cell Dev Biol 13: 395-424.

Branda SS, Gonzalez-Pastor JE, Ben-Yehuda S, Losick R, Kolter R. 2001. Fruiting body formation by Bacillus subtilis. Proc Natl Acad Sci 98: 11621-11626.

Branda SS, Gonzalez-Pastor JE, Dervyn E, Ehrlich SD, Losick R, Kolter R. 2004. Genes involved in formation of structured multicellular communities by Bacillus subtilis. I Bacteriol 186: $3970-3979$.

Branda SS, Chu F, Kearns DB, Losick R, Kolter R. 2006. A major protein component of the Bacillus subtilis biofilm matrix. Mol Microbiol 59: 1229-1238.

Chai Y, Chu F, Kolter R, Losick R. 2008. Bistability and biofilm formation in Bacillus subtilis. Mol Microbiol 67: 254-263.

Chai Y, Kolter R, Losick R. 2009. Paralogous antirepressors acting on the master regulator for biofilm formation in Bacillus subtilis. Mol Microbiol 74: 876-887.

Chen R, Guttenplan SB, Blair KM, Kearns DB. 2009. Role of the $\sigma^{\mathrm{D}}$-dependent autolysins in Bacillus subtilis population heterogeneity. I Bacteriol 191: 5775-5784.

Chu F, Kearns DB, Branda SS, Kolter R, Losick R. 2006. Targets of the master regulator of biofilm formation in Bacillus subtilis. Mol Microbiol 59: 1216-1228.

Chu F, Kearns DB, McLoon A, Chai Y, Kolter R, Losick R. 2008. A novel regulatory protein governing biofilm formation in Bacillus subtilis. Mol Microbiol 68: 1117-1127.

Cozy LM, Kearns DB. 2010. Gene position in a long operon governs motility development in Bacillus subtilis. Mol Microbiol (in press).

Errington J, Daniel RA, Scheffers D-J. 2003. Cytokinesis in bacteria. Microbiol Mol Biol Rev 67: 52-65.

Fujita M, Gonzalez-Pastor JE, Losick R. 2005. High- and Lowthreshold genes in the Spo0A regulon of Bacillus subtilis. I Bacteriol 187: 1357-1368.

Gaur NK, Cabane K, Smith I. 1988. Structure and expression of the Bacillus subtilis sin operon. I Bacteriol 170: 10461053.

Goehring NW, Beckwith J. 2005. Diverse paths to midcell: Assembly of the bacterial cell division machinery. Curr Biol 15: $514-526$.

Helmann JD, Marquez LM, Chamberlin MJ. 1988. Cloning, sequencing, and disruption of the Bacillus subtilis $\sigma^{28}$ gene. I Bacteriol 170: 1568-1574.

Kearns DB, Losick R. 2005. Cell population heterogeneity during growth of Bacillus subtilis. Genes \& Dev 19: 3083 3094.

Kearns DB, Chu F, Branda SS, Kolter R, Losick R. 2005. A master regulator for biofilm formation by Bacillus subtilis. Mol Microbiol 55: 739-749.
Kobayashi K. 2007. Bacillus subtilis pellicle formation proceeds through genetically defined morphological changes. J Bacteriol 189: 4920-4931.

Kobayashi K. 2008. SlrR/SlrA controls the initiation of biofilm formation in Bacillus subtilis. Mol Microbiol 69: 13991410.

Kuroda A, Sekiguchi J. 1993. High-level transcription of the major Bacillus subtilis autolysin operon depends on expression of the $\sigma^{\mathrm{D}}$ gene and is affected by a $\sin (f 1 a D)$ mutation. J Bacteriol 175: 795-801.

LaVallie ER, Stahl ML. 1989. Cloning of the flagellin gene from Bacillus subtilis and complementation studies of an in vitroderived deletion mutation. J Bacteriol 171: 3085-3094.

Lewis RJ, Brannigana JA, Smith I, Wilkinson AJ. 1996. Crystallisation of the Bacillus subtilis sporulation inhibitor SinR, complexed with its antagonist, Sinl. FEBS Lett 378: 98-100.

Lewis RJ, Brannigan JA, Offen WA, Smith I, Wilkinson AJ. 1998. An evolutionary link between sporulation and prophage induction in the structure of a repressor:anti-repressor complex. J Mol Biol 283: 907-912.

López D, Vlamakis H, Kolter R. 2009. Generation of multiple cell types in Bacillus subtilis. FEMS Microbiol Rev 33: 152163.

Maamar H, Dubnau D. 2005. Bistability in the Bacillus subtilis $\mathrm{K}$-state (competence) system requires a positive feedback loop. Mol Microbiol 56: 615-624.

Maamar H, Raj A, Dubnau D. 2007. Noise in gene expression determines cell fate in Bacillus subtilis. Science 317: 526529.

Margolin W. 2005. FtsZ and the division of prokaryotic cells and organelles. Nat Rev Mol Cell Biol 6: 862-871.

Margot P, Lazarevic V, Karamata D. 1996. Effect of the SinR protein on the expression of the Bacillus subtilis 168 lyt $A B C$ operon. Microb Drug Resist 2: 119-121.

Margot P, Pagni M, Karamata D. 1999. Bacillus subtilis 168 gene lyt $F$ encodes a $\gamma$-D-glutamate-meso-diaminopimelate muropeptidase expressed by the alternative vegetative $\sigma$ factor, $\sigma^{\mathrm{D}}$. Microbiology 145: 57-65.

Marquez LM, Helmann JD, Ferrari E, Parker HM, Ordal GW, Chamberlin MJ. 1990. Studies of $\sigma^{\mathrm{D}}$-dependent functions in Bacillus subtilis. J Bacteriol 172: 3435-3443.

Mirel DB, Chamberlin MJ. 1989. The Bacillus subtilis flagellin gene (hag) is transcribed by the $\sigma^{28}$ form of RNA polymerase. J Bacteriol 171: 3095-3101.

Mirel DB, Lauer P, Chamberlin MJ. 1994. Identification of flagellar synthesis regulatory and structural genes in a $\sigma^{\mathrm{D}}$-dependent operon of Bacillus subtilis. I Bacteriol 176: 4492-4500.

Murray EJ, Strauch MA, Stanley-Wall NR. 2009. $\sigma^{\mathrm{X}}$ is involved in controlling Bacillus subtilis biofilm architecture through the AbrB homologue Abh. J Bacteriol 191: 6822-6832.

Ohnishi R, Ishikawa S, Sekiguchi J. 1999. Peptidoglycan hydrolase LytF plays a role in cell separation with CwlF during vegetative growth of Bacillus subtilis. J Bacteriol 181: 31783184.

Oppenheim AB, Kobiler O, Stavans J, Court DL, Adhya S. 2005. Switches in bacteriophage $\lambda$ development. Annu Rev Genet 39: 409-429.

Piggot PJ, Hilbert DW. 2004. Sporulation of Bacillus subtilis. Curr Opin Microbiol 7: 579-586.

Pooley HM, Karamata D. 1984. Genetic analysis of autolysindeficient and flagellaless mutants of Bacillus subtilis. J Bacteriol 160: 1123-1129.

Smith TJ, Blackman SA, Foster SJ. 2000. Autolysins of Bacillus subtilis: Multiple enzymes with multiple functions. Microbiology 146: 249-262. 
Sonenshein AL. 2000. Control of sporulation initiation in Bacillus subtilis. Curr Opin Microbiol 3: 561-566.

Veening J-W, Smits WK, Kuipers OP. 2008. Bistability, epigenetics, and bet-hedging in bacteria. Annu Rev Microbiol 62: 193-210.

Vlamakis H, Aguilar C, Losick R, Kolter R. 2008. Control of cell fate by the formation of an architecturally complex bacterial community. Genes \& Dev 22: 945-953.

Vollmer W, Joris B, Charlier P, Foster S. 2008. Bacterial peptidoglycan (murein) hydrolases. FEMS Microbiol Rev 32: 259286. 


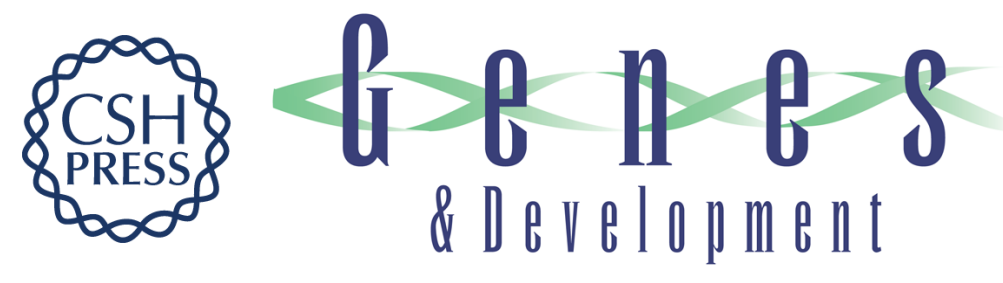

\section{An epigenetic switch governing daughter cell separation in Bacillus subtilis}

Yunrong Chai, Thomas Norman, Roberto Kolter, et al.

Genes Dev. 2010, 24: originally published online March 29, 2010

Access the most recent version at doi:10.1101/gad.1915010

\section{Supplemental http://genesdev.cshlp.org/content/suppl/2010/03/19/gad.1915010.DC1 \\ Material}

Related Content Swim or chill: lifestyles of a bacillus

David Dubnau

Genes Dev. April , 2010 24: 735-737

References This article cites 43 articles, 20 of which can be accessed free at: http://genesdev.cshlp.org/content/24/8/754.full.html\#ref-list-1

Articles cited in:

http://genesdev.cshlp.org/content/24/8/754.full.htmI\#related-urls

\section{License}

Email Alerting Receive free email alerts when new articles cite this article - sign up in the box at the top Service right corner of the article or click here.

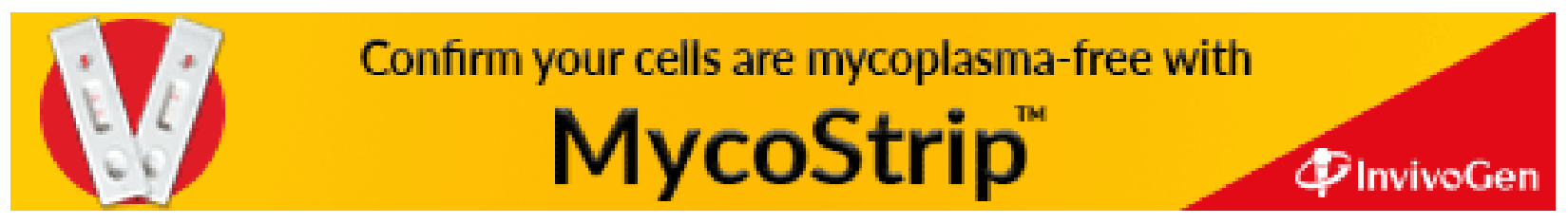

